\title{
Foreword for the Special issue on CMMSE 2017
}

\author{
Jesús Vigo Aguiar ${ }^{1}$
}

Published online: 5 June 2018

(C) Springer International Publishing AG, part of Springer Nature 2018

I am honoured to present this issue of selected papers representing some of the topics at the 17th International Conference on Computational and Mathematical Methods in Science and Engineering (CMMSE2017), held at Hotel Barcelo in Rota, Cadiz, Spain, in July 2017. The articles included in this issue provide additional detail and more extensive background information, than the related lectures presented at the conference. The papers collected here focus on two aspects:

(a) new advances in the theory of mathematical modelling of chemical phenomena, and

(b) the developments of new mathematical algorithms for computational techniques with applications in solving chemical problems.

The motivation for the selection of these topics has been to enhance the connections between the theoretical and some of the applied, practical aspects of the fundamental concepts of mathematical chemistry. We have attempted to cover topics and applications in rather diverse areas of mathematical chemistry, including basic physical concepts as applied to chemistry, and new algorithmic advances as to applied fields, such as drug discovery, biology, and chemistry-informatics.

It is my hope that our readers will find this collection of papers useful and motivating in furthering their own research in mathematical chemistry.

Please note that the JOMC paper, "A mathematical model for the pre-diagnostic of glioma growth based on blood glucose levels-CMMSE" by Luis Enrique Ayala

$凶$ Jesús Vigo Aguiar

jvigo@usal.es

1 Department of Applied Mathematics, Faculty of Science, University of Salamanca, 37008 Salamanca, Spain 
Hernández (MEXICO), published earlier in our journal, may also be considered as a part of this collection. 